\title{
Avaliação de tuberculose em crianças e adolescentes no Pará
}

\author{
Evaluation of tuberculosis in children and adolescents in Pará \\ Evaluación de tuberculosis en niños y adolescentes en Pará
}

Cristine Bessa Gondim ${ }^{1 *}$, Caroline Cunha da Rocha ${ }^{1}$, Isabela Cunha Oliveira de Vasconcellos ${ }^{3}$, Rafael Marcos Souza Ortiz ${ }^{2}$, Maiara Moraes Loureiro do Amaral ${ }^{2}$, Julieth Ferreira Sousa ${ }^{1}$, Leonardo Mota Aguiar Milhomem ${ }^{3}$, Thalita Cristina de Oliveira Brandão Campos ${ }^{1}$, Guilherme Alves da Silva ${ }^{4}$, Ana Claudia Alves Damasceno'.

\section{RESUMO}

Objetivo: $O$ trabalho propõe-se estudar a situação epidemiológica de casos novos da tuberculose entre a faixa etária de 0 a 19 anos no Estado do Pará, Brasil, no período de 2008 a 2018. Métodos: Trata-se de um estudo observacional descritivo, retrospectivo, de base de dados secundários, utilizando-se o Banco de dados do Sistema de Informações de Agravos de Notificação (SINAN). Avaliando as seguintes variáveis: idades, sexo, forma clínica da doença, forma diagnóstica e desfecho dos casos. Resultados: O número absoluto de pacientes triados foi de 4452 pacientes. Verificou-se o coeficiente de incidência acima da média $5,6 \%$ durante o período. Houve predomínio do sexo masculino. A forma clínica pulmonar ocorreu em $84,35 \%$ dos casos. A maioria dos casos $(69,9 \%)$ evoluíram para a cura. Conclusão: Os resultados apresentados contribuem para o conhecimento da situação epidemiológica da tuberculose, fornecendo subsídios à tomada de decisões relacionadas principalmente às ações de prevenção e controle da doença no Estado do Pará.

Palavras-chave: Tuberculose, Pediatria, Epidemiologia.

\begin{abstract}
Objective: This paper aims to study the epidemiological situation of new tuberculosis cases between the age group of 0 to 19 years in the State of Pará, Brazil, from 2008 to 2018. Methods: This is a descriptive observational study. retrospective, secondary database, using the Notification Disease Information System Database (SINAN). Evaluating the following variables: ages, gender, clinical form of the disease, diagnostic form and outcome of the cases. Results: The absolute number of screened patients was 4452 patients. The incidence coefficient above the average was $5.6 \%$ during the period. There was a predominance of males. The pulmonary clinical form occurred in $84.35 \%$ of the cases. Most cases $(69.9 \%)$ evolved to cure. Conclusion: The results presented contribute to the knowledge of the epidemiological situation of tuberculosis, providing support to decision making related mainly to the prevention and control of the disease in the State of Pará.
\end{abstract}

Key words: Tuberculosis, Pediatrics, Epidemiology.

\section{RESUMEN}

Objetivo: este trabajo tiene como objetivo estudiar la situación epidemiológica de los nuevos casos de tuberculosis entre el grupo de edad de 0 a 19 años en el estado de Pará, Brasil, de 2008 a 2018. Métodos: Este es un estudio observacional descriptivo. base de datos secundaria retrospectiva, utilizando la base de

\footnotetext{
1Universidade Federal do Pará. Belém-Pará. *E-mail: dra_cristine@yahoo.com.br

${ }^{2}$ Hospital de Pronto Socorro Municipal Mario Pinotti. Belém-Pará.

${ }^{3}$ Centro Universidade do Pará. Belém-Pará.

${ }^{4}$ Centro Universitário Metropolitano da Amazônia. Belém-Pará.
} 
datos del Sistema de Información de Enfermedades de Notificación (SINAN). Evaluación de las siguientes variables: edad, sexo, forma clínica de la enfermedad, forma diagnóstica y resultado de los casos. Resultados: El número absoluto de pacientes seleccionados fue de 4452 pacientes. El coeficiente de incidencia superior a la media fue del 5,6\% durante el período. Predominó el sexo masculino. La forma clínica pulmonar se produjo en el $84,35 \%$ de los casos. La mayoría de los casos $(69,9 \%)$ evolucionaron para curarse. Conclusión: Los resultados presentados contribuyen al conocimiento de la situación epidemiológica de la tuberculosis, brindando apoyo para la toma de decisiones relacionadas principalmente con la prevención y el control de la enfermedad en el Estado de Pará.

Palabras clave: Tuberculosis, Pediatría, Epidemiología.

\section{INTRODUÇÃO}

A tuberculose (TB), segundo Brasil (2018), é a principal doença infecto-contagiosa de origem bacteriana no mundo. Aproximadamente dois bilhões de pessoas estão infectadas pelo agente Mycobacterium Tuberculosis e, dentre essas, oito milhões desenvolverão a doença e dois milhões morrerão por ano. O principal reservatório da TB é o homem sendo transmitida de pessoa a pessoa por meio do de aerossóis. $O$ espirro e a tosse de um paciente tuberculoso bacilífero lança no ar gotículas contendo no seu interior o bacilo. Quanto ao período de incubação, após a infecção, perpassam de 4 a 12 semanas para a detecção dos sintomas iniciais. A maioria dos novos casos de doença pulmonar ocorre em torno de 12 meses após a infecção inicial (ARAUJO SRL, et al.,2015).

O Brasil está entre os 20 países com maior número de casos da patologia, sendo responsável por $82 \%$ dos casos mundiais e $75 \%$ dos casos envolvendo crianças. Há cerca de 1 milhão de casos na população infantil do mundo, e a doença é responsável por 130 mil mortes por ano. No Brasil, de acordo com o Sistema de Informação de Agravos de Notificação (SINAN), em 2015, foram registrados 83.617 casos de TB, dos quais 7.106 ocorreram em menores de 19 anos de idade. Os casos pediátricos da doença chegam a representar $10 \%$ dos casos da doença (BRASIL, 2018).

A taxa de mortalidade entre crianças não tratadas é estimada em $21,9 \%$. No entanto, foi relatado que essa mortalidade pode ser reduzida quando o tratamento é realizado de forma continuada. A mortalidade por tuberculose em crianças é subestimada, pois, para muitas crianças que morrem por tuberculose, a causa da morte é listada como outras patologias de base como pneumonia (CARVALHO ACC, et al., 2018). Em relação a história natural da doença esse grupo etário apresenta elevado risco de progressão para a doença ativa e maior risco de desenvolvimento de formas extrapulmonares. A incidência incide principalmente em menores de 5 anos de idade, e as crianças se infectam após aproximadamente 20 minutos de exposição ao agente infeccioso (CARVALHO ACC, et al., 2018).

O quadro clínico de tuberculose na infância apresenta sinais e sintomas inespecíficos e algumas crianças ainda são assintomáticas. Dentre os sintomas mais comuns estão: febre, perda de apetite, sudorese noturna, perda de peso e dor torácica. Outros sinais e sintomas incluem palidez, linfadenopatia, hepatoesplenomegalia, eritema nodoso, ceratoconjuntivite e dor articular. A tosse é o principal sintoma da forma pulmonar da doença, que é a forma mais comum de tuberculose pediátrica (CARVALHO ACC, et al., 2018).

$O$ grande desafio relacionado à TB em crianças é o diagnóstico. As técnicas diagnósticas utilizadas em adultos apresentam baixa sensibilidade e especificidade em crianças (CANO APG, et al., 2017). Já ao término da infância e início da adolescência, aparecem formas semelhantes às encontradas em adultos, visto que as pessoas quase sempre têm sintomas respiratórios e os resultados positivos à baciloscopia são frequentes (ROSSONI AMO, ROSSONI MD, RODRIGUE CO, 2013).

O principal consenso internacional sobre tuberculose na infância traz a noção da dificuldade diagnóstica e enorme quantidade de subnotificação na população devido os fracos indicíos da patologia, seja características clínicas, radiológicas e epidemiológicas. Assim, a conduta para o diagnóstico em crianças se baseia nos aspectos: história clínica (dando ênfase para história de contato); exame clínico; resultado da 
Prova Tuberculínica (PT), achados da radiografia de tórax, dentre outros (OMS, 2017). Assim, o presente trabalho buscou identificar as crianças e adolescentes diagnosticados com tuberculose no Estado do Pará do período de 2008 a 2018. Buscou-se identificar assim as formas de diagnóstico e as formas clínicas apresentadas na região. Esse estudo tem como princípio complementar o banco de dados nacional para que a comunidade científica e a população possam dar importância ao assunto supracitado. E assim, evitar as possíveis complicações da doença para a população pediátrica e as subnotificações que são tão prevalentes.

\section{MÉTODOS}

O estudo foi desenvolvido por meio da análise de dados do Estado do Pará analisando a população em relação ao perfil epidemiológico da Tuberculose no período de 2008 a 2018, durando o período de 3 meses para a coleta adequada, realizado pelos autores da pesquisa.

O tipo do estudo foi observacional descritivo de âmbito populacional usando fonte de dados extraídos do Sistema de Informação de Agravos de Notificação SINAN, no qual, os profissionais são capacitados para tal atividade, de acordo com as variáveis encontradas na ficha de notificação de TB. Foram incluídos no estudo varáveis: demográficas: sexo e grupo etário; clínicas: forma clínica; métodos diagnósticos: exame de baciloscopia e exame de cultura; situação de encerramento do caso: cura, abandono, óbito por TB.

A análise no software Microsoft Excel 2010. As variáveis quantitativas foram descritas por meio de seu valor absoluto, de distribuição de frequências e da taxa de ocorrência. A presente pesquisa estará de acordo com os preceitos da Declaração de Helsinque e do Código de Nuremberg, respeitando as Normas de Pesquisa Envolvendo Seres Humanos (Res. 466/12) do Conselho Nacional de Saúde e ocorrerá após o aceite do orientador responsável pelo estudo.

\section{RESULTADOS}

No presente estudo, as variáveis obtidas demonstraram, em sua maioria, resultados que corroboração com demais artigos encontrados na literatura acerca do tema. Entretanto, algumas discordâncias apontam para a necessidade de mais estudos sobre esta patologia, buscando-se abranger as diversas realidades que a permeiam, assim como esta pesquisa busca cumprir.

De acordo com a (Tabela 1), a quantidade de casos confirmados de TB em crianças e adolescentes no período de 2008 a 2018 foi de 4552 . Numa proporção de 1435 (31,52\%) crianças de 0 a 14 anos para 3117 $(31,52 \%)$ adolescentes de 15 a 19 anos. Percebe-se ainda por meio da tabela que o maior ano de prevalência de diagnóstico foi no ano de 2017 (491) e o menor em 2018 (328) mostrando uma decaída de um ano ao outro.

Tabela 1 - Distribuição do número e frequência de casos novos de tuberculose por grupo etário e ano do diagnóstico, na população pediátrica e jovem do Estado do Pará - 2008 a 2018.

\begin{tabular}{lllllllllllll}
\hline Ano & \multicolumn{1}{c}{} & \multicolumn{3}{c}{$1-4$} & \multicolumn{3}{c}{$5-9$} & $10-14$ & \multicolumn{3}{c}{$15-19$} & \multicolumn{3}{c}{ Total } \\
\hline & $\mathrm{N}$ & $\%$ & $\mathrm{~N}$ & $\%$ & $\mathrm{~N}$ & $\%$ & $\mathrm{~N}$ & $\%$ & $\mathrm{~N}$ & $\%$ & $\mathrm{~N}$ & $\%$ \\
\hline $\mathbf{2 0 0 8}$ & 11 & 2,76 & 26 & 6,53 & 27 & 6,78 & 64 & 16,0 & 270 & 67,83 & 398 & 8,74 \\
\hline $\mathbf{2 0 0 9}$ & 28 & 6,42 & 33 & 7,56 & 23 & 5,27 & 80 & 18,34 & 272 & 62,38 & 436 & 9,57 \\
\hline $\mathbf{2 0 1 0}$ & 25 & 5,81 & 26 & 6,04 & 22 & 5,11 & 60 & 13,95 & 297 & 69,07 & 430 & 9,44 \\
\hline $\mathbf{2 0 1 1}$ & 24 & 5,24 & 26 & 5,67 & 25 & 5,45 & 72 & 15,72 & 311 & 67,90 & 458 & 10,0 \\
\hline $\mathbf{2 0 1 2}$ & 16 & 3,98 & 27 & 6,71 & 29 & 7,21 & 47 & 11,69 & 283 & 70,39 & 402 & 8,83 \\
\hline $\mathbf{2 0 1 3}$ & 22 & 5,33 & 23 & 5,58 & 15 & 3,64 & 72 & 17,47 & 280 & 67,96 & 412 & 9,05 \\
\hline $\mathbf{2 0 1 4}$ & 18 & 4,76 & 15 & 3,96 & 25 & 6,61 & 55 & 14,55 & 265 & 70,10 & 378 & 8,30 \\
\hline $\mathbf{2 0 1 5}$ & 15 & 3,98 & 15 & 3,98 & 12 & 3,19 & 59 & 15,69 & 275 & 73,13 & 376 & 8,26 \\
\hline $\mathbf{2 0 1 6}$ & 24 & 5,41 & 33 & 7,44 & 19 & 4,28 & 61 & 13,76 & 306 & 69,07 & 443 & 9,73 \\
\hline $\mathbf{2 0 1 7}$ & 37 & 7,53 & 35 & 7,12 & 15 & 3,05 & 64 & 13,03 & 340 & 69,24 & 491 & 10,78 \\
\hline $\mathbf{2 0 1 8}$ & 21 & 6,40 & 26 & 7,92 & 20 & 6,09 & 43 & 13,10 & 218 & 66,46 & 328 & 7,20 \\
\hline Total & 241 & & 285 & & 232 & & 677 & & 3117 & & 4552 & 100 \\
\hline
\end{tabular}

Fonte: Ministério da Saúde - Sistema de Informação de Agravos de Notificação (SINAN), 2008 a 2018. 
Quanto a progressão anual dentre os 11 anos analisados observou-se o primeiro aumento expressivo de 2015 (n: 376) a 2017 (n:491) e uma queda acentuada do ano de 2017 a 2018 (n:328). Segundo Carvalho et al., (2018) o entendimento dos pais sobre a doença devido a propagação de saúde e educação auxilia nessa procura dos centros de saúde, associado ainda a maior facilidade do diagnóstico. E a queda, pode ser explicada pelo subdiagnóstico ou o não preenchimento adequado do SINAN.

$\mathrm{Na}$ (Tabela 2) verificam-se coeficientes de incidência de casos novos de TB em menores de 20 anos no período de estudo, e um coeficiente de incidência médio 5.6 por 100.000 , e é possível identificar ainda que os coeficientes se mantem estáveis por todos os anos, sem queda ou aumento expressivo. A população utilizada nos anos de 2008 e 2009, segundo IBGE de 2000 foi de 6.192.307 habitantes no estado do Pará. Já a população nos anos subsequentes até o ano de 2018 segundo IBGE de 2010, foi de 7.581.051. Não se utilizou a população estimada em nenhum dos anos colhidos.

Tabela 2 - Coeficiente de incidência de casos novos de tuberculose em crianças e adolescentes por 100.000 habitantes, no Estado do Pará - 2008 a 2018.

\begin{tabular}{cccc}
\hline \multicolumn{5}{c}{ Número de casos } & Coeficiente de Incidência & \\
\hline ANO & $(\mathrm{N})$ & $($ Nx100.000/População & População \\
\hline $\mathbf{2 0 0 8}$ & 398 & 6.4 & 6192307 \\
\hline $\mathbf{2 0 0 9}$ & 436 & 7 & 6192307 \\
\hline $\mathbf{2 0 1 0}$ & 430 & 5.6 & 7581051 \\
\hline $\mathbf{2 0 1 1}$ & 458 & 6 & 7581051 \\
\hline $\mathbf{2 0 1 2}$ & 402 & 5.3 & 7581051 \\
\hline $\mathbf{2 0 1 3}$ & 412 & 5.4 & 7581051 \\
\hline $\mathbf{2 0 1 4}$ & 378 & 4.9 & 7581051 \\
\hline $\mathbf{2 0 1 5}$ & 376 & 4.9 & 7581051 \\
\hline $\mathbf{2 0 1 6}$ & 443 & 5.8 & 7581051 \\
\hline $\mathbf{2 0 1 7}$ & 491 & 6.4 & 7581051 \\
\hline $\mathbf{2 0 1 8}$ & 328 & 4.3 & 7.328 .552 \\
\hline Média & 413.8 & 5.6 & \\
\hline
\end{tabular}

Fonte: Ministério da Saúde - Sistema de Informação de Agravos de Notificação (SINAN), 2008 a 2018.

A (Tabela 3) mostra que houve predomínio do sexo masculino, superior a $50 \%$ no total. Exceto em 2009 com maior percentual no sexo feminino. Tendo um total de $2519(55,3 \%)$ no sexo masculino e $2033(44,6 \%)$ no sexo feminino.

Tabela 3 - Distribuição do número de casos novos de tuberculose por sexo, na população pediátrica e jovem do Estado do Pará - 2008 a 2018.

\begin{tabular}{lccccccccccc}
\hline Ano & 2008 & 2009 & 2010 & 2011 & 2012 & 2013 & 2014 & 2015 & 2016 & 2017 & 2018 \\
\hline Masculino & 214 & 212 & 256 & 261 & 209 & 232 & 211 & 193 & 269 & 271 & 191 \\
\hline Feminino & 184 & 224 & 174 & 197 & 193 & 180 & 167 & 183 & 174 & 220 & 137 \\
\hline Total & 398 & 436 & 430 & 458 & 402 & 412 & 378 & 376 & 443 & 491 & 328 \\
\hline
\end{tabular}

Fonte: Ministério da Saúde - Sistema de Informação de Agravos de Notificação (SINAN), 2008 a 2018.

A amostra total de pacientes mostrou uma elevada presença de tuberculose - seja pulmonar ou extrapulmonar - nos pacientes pediátricos (0-14 anos) e nos jovens (15-19 anos). Tendo um total de 1435 (31,52\%) pacientes entre 0 e 14 anos (pediátricos) e 3117 (68,47\%) entre 15 e 19 anos (adolescentes). Possível relacionar essa pesquisa com o estudo de Marques (2014), que analisou pacientes até 15 anos no período de 7 anos, tendo um valor expressivo também como resultado: 3734 pacientes.

$\mathrm{Na}$ Tabela 4 observa-se a forma clínica prevalente em cada faixa etária. Tendo a forma pulmonar predominando em todas as situações (total de $84,35 \%$ do total). 
Tabela 4 - Distribuição do número de casos novos de tuberculose por faixa etária segundo a forma clínica em pacientes pediátricos e jovens no Pará - 2008 a 2018.

\begin{tabular}{ccccccc}
\hline Forma clínica & $<1$ & $1-4$ & $5-9$ & $10-14$ & $15-19$ & Total \\
\hline Pulmonar & 199 & 176 & 132 & 507 & 2826 & 3840 \\
\hline Extrapulmonar & 25 & 75 & 63 & 140 & 291 & 594 \\
\hline Pulmonar+Extrapulmonar & 02 & 20 & 20 & 23 & 53 & 118 \\
\hline Total & 226 & 271 & 215 & 670 & 3170 & 4552 \\
\hline
\end{tabular}

Fonte: Ministério da Saúde - Sistema de Informação de Agravos de Notificação (SINAN), 2008 a 2018.

$\mathrm{Na}$ (Tabela 5) mostra a frequência de diagnostico realizada nos pacientes da pesquisa em questão a baciloscopia.

Tabela 5 - Realização de baciloscopia nos pacientes pediátricos e jovens no Estado do Pará - 2008 a 2018.

\begin{tabular}{ccccccc}
\hline Total & $<1$ & $1-4$ & $5-9$ & $10-14$ & $15-19$ & Total \\
\hline Positivo & 108 & 26 & 33 & 315 & 2129 & 2611 \\
\hline Negativo & 57 & 41 & 52 & 172 & 648 & 970 \\
\hline Sem dados & 76 & 218 & 147 & 190 & 340 & 971 \\
\hline Total & 241 & 285 & 232 & 677 & 3117 & 4552
\end{tabular}

Fonte: Ministério da Saúde - Sistema de Informação de Agravos de Notificação (SINAN), 2008 a 2018.

A (Tabela 6) expressa a situação de encerramento dos casos de TB por ano. No total de casos avaliados, $69,9 \%$ evoluíram para cura, $1 \%$ para óbito por outras causas ( 47 casos), $8,3 \%$ por abandono.

Tabela 6 - Situação de encerramento de casos de tuberculose em pacientes pediátricos e jovens no Pará - 2008 a 2018.

\begin{tabular}{lcccccc}
\hline Total & $<1$ & $1-4$ & $5-9$ & $10-14$ & $15-19$ & Total \\
\hline Cura & 148 & 179 & 162 & 477 & 2218 & 3184 \\
\hline Aband & 13 & 8 & 19 & 42 & 297 & 379 \\
\hline Óbito & 9 & 7 & 5 & 5 & 16 & 42 \\
\hline Ó.O.C & 8 & 9 & 3 & 6 & 21 & 47 \\
\hline TB-DR & 29 & 43 & 14 & 59 & 199 & 344 \\
\hline M.D & 1 & 1 & - & 2 & 8 & 12 \\
\hline A.P & - & 1 & 1 & - & 4 & 6 \\
\hline N.R & 33 & 37 & 28 & 86 & 354 & 538 \\
\hline Total & 241 & 285 & 232 & 677 & 3117 & 4552 \\
\hline
\end{tabular}

Legenda: Aband: Abandono; O.O.C: Óbito por outras causas; TB-DR: Tuberculose droga resistente; MD: Mudança de diagnóstico; AP: Abandono primário; NR: Não realizado.

Fonte: Ministério da Saúde - Sistema de Informação de Agravos de Notificação (SINAN), 2008 a 2018.

\section{DISCUSSÃO}

O coeficiente de incidência de TB teve uma média 5,6 por 100.000 habitantes verificados no estudo. É uma alta taxa e concordante com as encontradas em grupos em desvantagens social, em consonância à comunidade com baixa incidência cujos coeficientes variam de 1 a 10/100.000 (SANTOS M, 2012). No estudo de Venâncio TS, Tuan TS, Nascimento LFC (2015) foram identificados 2513 casos novos de tuberculose na população até 15 anos, com uma incidência de 2,13 casos/100.000 habitantes e, ainda, no estudo de Marques KM (2014) mostrou uma incidência de 1,4 casos/100.000. Confirmando assim, a alta incidência no Estado do Pará no período da pesquisa, sendo ainda mais alta que os estudos supracitados.

Segundo estimativas da OMS (2017), o Brasil tem uma incidência anual de 43 casos por 100 mil habitantes, uma taxa de incidência da forma pulmonar positiva de 26/100 mil habitantes. Concluindo assim que a taxa de incidência de tuberculose nos pacientes pediátricos e jovens no Estado do Pará está elevada 
em relação preconizada, levando em consideração o tempo do estudo e a população analisada. Pinto JTJM (2017) reafirma a elevada incidência em nosso Estado, afirmando que as regiões nortes e sudestes lideram como os estados com maior número de casos por mil habitantes, o primeiro com 37,4 e o segundo com 34,1 .

Outro fator tangível é citado por Lima SS, et al., (2017) que realizou seu estudo no Estado do Pará. O estudo afirma que a TB tem relação com variáveis sociais e demonstra relação direta com indicadores de exclusão social. Ocorre ainda associação da TB com fatores socioeconômicos e concluem a existência de dependência da TB com a situação social da população.

Em relação a prevalência de sexo, 55,3\% dos pacientes até 20 anos são do sexo masculino, concordando com Marques KM (2014) que teve mais de 50\% dos pacientes pediátricos sendo do sexo masculino; ainda concorda com Dias BAS, et al., (2014) e está em discordância com o estudo de Cano APG, et al, (2017). Os estudos avaliados não abordam o motivo da prevalência de um sexo em detrimento do outro, já que as variáveis socioeconômicas estão diretamente relacionadas a patologia estudada.

Quanto à forma clínica, $84,35 \%$ dos pacientes apresentaram a forma pulmonar, e os que apresentam forma extrapulmonar houve o predomínio da forma ganglionar (12 pacientes). Estes dados estão em consonância com os principais estudos comparados: Marques KM (2014); Cano APG, et al, (2017); Dias BAS, et al., (2014); e Venâncio TS, Tun TS, Nascimento LFC (2015) em relação a predominância da forma pulmonar. Esses estudos afirmam que ocorre o predomínio da forma pulmonar da doença no grupo etário dos pacientes com menos de cinco anos, característica de ambiente com alta incidência de TB - que é o caso do Estado do Pará - e manutenção da cadeira de transmissão da doença. Quanto à forma extrapulmonar, observou-se que a falta de dados concisos atrapalha a veracidade dessa variável. Com os poucos dados encontrados, a forma ganglionar foi a predominante (12 pacientes), seguido da forma óssea (3 pacientes) e forma miliar (2). No estudo de Cano APG et al., (2017) a predominância de TB óssea ocorreu em pacientes menores de 10 anos e esse tipo extrapulmonar foi o prevalente.

Interessante ainda citar que as formas pulmonares inúmeras vezes se sobrepõem a forma extrapulmonar - que no presente estudo foi o caso de 2,59\% dos pacientes - e segundo Pinto JTJM (2017) essa correlação é usual quando diagnosticado corretamente.

Em relação ao diagnóstico de tuberculose na infância e adolescência, nas duas últimas décadas, verificaram-se avanços significativos nesta área, mas muitas das novas técnicas de diagnóstico ou não foram validadas na população pediátrica ou não são acessíveis nas regiões endémicas de tuberculose (REI SVM, 2017). O presente estudo avaliou a frequência de realização de dois principais exames: cultura e baciloscopia. Em $78,6 \%$ ( $n=3571$ ) dos pacientes foi realizado baciloscopia, tendo resultado positivo em $57,3 \%$ dos casos e negativo em $21,3 \%$. Já em relação a cultura, foi realizada em $8,8 \%(n=401)$ dos pacientes, sendo positiva em $5,71 \%$ e negativa em $3,1 \%$. Notável a valorização e disponibilidade maior do primeiro método diagnóstico, no entanto Rei SVM (2017) afirma que o isolamento de M. tuberculosis em cultura é considerado o método padrão-ouro para o diagnóstico definitivo de tuberculose em crianças sintomáticas. E esse método tem no custo uma de seus principais entraves.

No estudo de Marques KM (2014) 38,1\% das crianças realizaram primeira baciloscopia direta de escarro para pesquisa de bacilo álcool-ácido (BAAR) com positividade de $24,4 \%$. Já a cultura de escarro para bacilo de Koch (BK) foi realizada em $35,8 \%$ dos pacientes, com uma positividade de $44,4 \%$. O estudo de Cano APG, et al., (2017) mostra que a positividade da cultura e da baciloscopia foram, respectivamente, 65,8 e 35,7.

Rabahi MF, et al., (2017) e outras literaturas afirmam que, apesar de a pesquisa e a cultura de micobactéria serem os métodos diagnósticos mais utilizados quando existe suspeita de casos da patologia, menos da metade das crianças com o diagnóstico apresentam baciloscopia positiva e a cultura detecta M. tuberculosis em menos de $50 \%$ dos casos. Demonstrando que o contato com um adulto com tuberculose e a clínica do paciente pediátrico são de extrema importância no diagnóstico.

Assim, formular o diagnóstico de tuberculose na criança é algo que necessita de avaliação dos pormenores, onde as peças principais são os sinais e sintomas clínicos, as alterações radiológicas e a história epidemiológica. Em alguns casos, conta-se com o auxílio de peças extras, como exames laboratoriais (provas 
de atividade inflamatória, baciloscopia, culturas, exames moleculares, pesquisa de antígenos, IGRA e exame anatomopatológico) e a resposta clínica do paciente (ROSSONI AMO, ROSSONI MD, RODRIGUES CO, 2013).

Por fim, a taxa de cura dos pacientes pediátricos e jovens diagnosticados no período da pesquisa foi de $69,9 \%$, a taxa de abandono foi de $8,3 \%$ e de óbito $0,9 \%$. A taxa de cura não superou a meta de $85 \%$ pactuada pelo Programa Nacional de Tuberculose (PNTC) com OMS (SANTOS SC, 2015), devido abandono dos pacientes que muitas vezes não tem sintomas e leva a descontinuação da terapia adequada. Marques KM (2014) apresentou uma taxa de 74,3\%; Cano APG, et al., (2017) obteve um resultado de 82,8\% de cura; Dias BAS, et al., (2014) obteve $80,31 \%$. Tendo o aspecto negativo de que todos os estudos comparados na literatura não atingiram a meta de $85 \%$ do PNTC. Trazendo assim, a necessidade de acompanhamento de forma mais concisa os pacientes e seus pais alertando assim para os perigos da doença não tratada e as possíveis complicações.

\section{CONCLUSÃO}

Focado no diagnóstico, o estudo em questão observou o grande número de casos de tuberculose em crianças e adolescentes de 2008 a 2018, totalizando 4452 casos de crianças de $<1$ a 19 anos. Houve ainda a prevalência do sexo masculino; a forma pulmonar predominou sendo que a forma extrapulmonar mais comum foi a ganglionar. A partir desse estudou, notou-se uma real necessidade um estudo aprofundado acerca do assunto. O subdiagnóstico por vezes ocorre por conta da dificuldade diagnóstica na população pediátrica. Os desafios diagnósticos contribuem para a subestimação do impacto epidemiológico dessa patologia nessa faixa etária, que leva a uma menor prioritização pelos programas de saúde pública.

\section{REFERÊNCIAS}

1. ARAUJO SRL, et al. Perfil epidemiológico da tuberculose pulmonar na cidade do Natal RN. Official Journal of the Brazilian Association of Infection Control and Hospital Epidemiology. V4, p 1-4. 2015.

2. BRASIL. Ministério da Saúde. Secretaria de Vigilância em Saúde. Boletim Epidemiológico, v. 47, n.13, 2016.

3. BRASIL. Manual de Recomendações para o Controle da Tuberculose no Brasil. Ministério da Saúde, Secretaria de Vigilância em Saúde, Departamento de Vigilância das Doenças Transmissíveis. - Brasília: Ministério da Saúde, 2018.

4. CANO APG, et al. Tuberculose em pacientes pediátricos: como tem sido feito o diagnóstico? Rev. paul. pediatr, São Paulo, v. 35, n. 2, p. 165-170, June 2017.

5. CARVALHO ACC, et al. Aspectos epidemiológicos, manifestações clínicas e prevenção da tuberculose pediátrica sob a perspectiva da Estratégia End TB. J. bras. pneumol. São Paulo, v. 44, n. 2, p. 134-144, Apr. 2018.

6. CARVALHO ER, et al. Esquemas de tratamento da tuberculose na infância e eventos adversos relacionados. Resid Pediatr. 2018;8(1):20-26 DOI: 10.25060/residpediatr-2018.v8n1-03.

7. DIAS BAS, SALES CMM, BERTOLDE AI, MACIEL ELN. Análise espacial da tuberculose infantil no Espírito Santo no período de 2001 a 2011. Revista Brasileira de Pesquisa em Saúde/Brazilian Journal of Health Research, 16(3), 9298, 2014.

8. LIMA SS, et al. Análise espacial da tuberculose em Belém, estado do Pará, Brasil. Rev PanAmaz Saude. Ananindeua, v. 8, n. 2, p. 55-63, jun. 2017.

9. MARQUES KM. Perfil epidemiológico de casos novos de Tuberculose em menores de 15 anos no distrito federal 2002 a 2008. J. bras. Pneumol. Distrito Federal, v. 20, n. 1, p. 120-144, Apr. 2014

10. MENDONÇA AS, FRANCO SC. Avaliação do risco epidemiológico e do desempenho dos programas de controle de tuberculose nas Regiões de Saúde do estado de Santa Catarina, 2003 a 2010. Epidemiol. Serv. Saúde, v2, p 10-34, 2015.

11. ORGANIZAÇÃO MUNDIAL DE SAÚDE. Guidance for national tuberculosis programmes on the management of tuberculosis in children. 2ed. 2017.

12. PINHEIRO M, ARAUJO AR, GUEDES M. Tuberculose infantil: novas formas de diagnóstico. Nascer e Crescer, Porto, v. 24, n. 4, p. 160-165, dez. 2015.

13. PINTO JTJM. Acesso das pessoas menores de quinze anos com tuberculose aos servições de saúde. $2017.192 f$. Tese (Doutorado em Saúde Coletiva). Universidade Federal do Rio Grande do Norte, Natal. 
14. RABAHI MF, et al. Tuberculosis treatment. J Bras Pneumol, 2017.

15. REI SVM. Tuberculose Multirresistente em Idade Pediátrica: Diagnóstico e Tratamento. 2017. 80f. Tese (Mestrado em Saúde Coletiva). Universidade de Coimbra, Portugal.

16. ROSSONI AMO, ROSSONI MD, RODRIGUES CO. Critérios de pontuação para diagnóstico de tuberculose em crianças. Pulmão RJ, v.22, n.3, p. 65-69. 2013.

17. SANTOS M. Atlas nacional do Brasil Milton Santos. Rio de Janeiro: IBGE, 2012. 307 p.

18. SANTOS SC. Tuberculose em indigenas menores de 14 anos no Mato Grosso do Sul: aspectos epidemiologicos e desafio diagnostico - 2000 a 2010. Campo Grande (ms): Universidade Federal de Mato Grosso do Sul, 2015.

19. VENANCIO TS, TUAN TS, NASCIMENTO LFC. Incidência de tuberculose em crianças no estado de São Paulo, Brasil, sob enfoque espacial. Ciênc. saúde coletiva, Rio de Janeiro, v. 20, n. 5, p. 1541-1547, Maio, 2015. 